\title{
In vitro fermentation of raffinose to unravel its potential as prebiotic ingredient
}

\author{
Cláudia Amorim, Sara C. Silvério, Beatriz B. Cardoso, Joana I. Alves, Maria Alcina Pereira, \\ Lígia R. Rodrigues*
}

CEB-Centre of Biological Engineering, Universidade do Minho, Campus de Gualtar, 4710-057, Braga, Portugal

\section{A R T I C L E I N F O}

\section{Keywords:}

Prebiotic

Lactulose

Raffinose

Human fecal inocula

in vitro fermentation

\begin{abstract}
A B S T R A C T
Until now the prebiotic potential of pure trisaccharide raffinose on human health assessed through highthroughput sequencing remains poorly investigated. In this work, an in vitro model using human fecal inocula of two healthy volunteers (D1 and D2) was used to study the prebiotic potential of raffinose and compare it with the well-stablished and commercial prebiotic lactulose. The intestinal microbiota showed preference for raffinose as substrate showing the highest consumption value at $48 \mathrm{~h}(96.0 \pm 0.9 \% \mathrm{D} 1$ and $95.3 \pm 0.7 \% \mathrm{D} 2)$. The fermentation of raffinose decreased the medium $\mathrm{pH}$, the ammonia concentration and the relative amount of Proteobacteria, while increasing the total production of lactate and short chain fatty acids $(129.9 \pm 2.6 \mathrm{mmol} / \mathrm{L}$ $\mathrm{D} 1$ and $179.6 \pm 0.6 \mathrm{mmol} / \mathrm{L} \mathrm{D} 2), \mathrm{CO}_{2}\left(10.8 \pm 0.8 \mathrm{mmol} / \mathrm{L}_{\text {medium }} \mathrm{D} 1\right.$ and $\left.5.2 \pm 0.3 \mathrm{mmol} / \mathrm{L}_{\text {medium }} \mathrm{D} 2\right)$ and the relative amount of Bifidobacterium and Lactobacillus. This study suggests that raffinose holds potential functional properties for human health.
\end{abstract}

\section{Introduction}

The loss of food functional properties due to the current industrial processing and the growing changes on people modern lifestyles associated with unhealthy eating behaviors are among the negative consequences of the globalization impact (Adamberg et al., 2014). As a result, the global epidemic of diet-related diseases had become a major public health issue (WHO - World Health Organization, 2018). However, the consumer awareness has increased towards the importance of using food as preventive medication, being willing to pay more for healthier eating options (Forbes, 2015). Thus, bioactive compounds produced from a natural source are currently preferred to modern therapeutic agents (Samanta et al., 2015). In particular, the use of bioactive compounds, such as prebiotics, has been shown to be very attractive given their role on modulating the gut microbiota and the subsequent beneficial effects on human and animal health over different physiological functions. The link between gut microbiota health and several different disorders, e.g. disbyosis caused by antibiotics intake or food poisoning, has been the recent focus of increased research (Kang et al., 2019; Yeung, Mocan, \& Atanasov, 2018).

Recently updated by the International Scientific Association for Probiotics and Prebiotics (ISAPP), the current definition establishes a prebiotic as "a substrate that is selectively utilized by host microorganisms conferring a health benefit", and the host can be either human or animal (Gibson et al., 2017). Prebiotic beneficial effects have been reported on the gastrointestinal tract, cardiometabolism, skeletal system, nervous system, among others (Gibson et al., 2017; Saad, Delattre, Urdaci, Schmitter, \& Bressollier, 2013).

The global market demand of prebiotic is in expansion, registering 623.5 kton in 2015 and growing at a compound annual growth rate of 8.0\% from 2016 to 2024 (Grand View Research, 2018). Prebiotic carbohydrates, particularly oligosaccharides, are the most widely studied and the best market established type of prebiotics. These compounds are also marketed beyond their traditional functional properties, being industrially used for instance as sugar and fat replacement, as well as taste and texture enhancement (Das, Choudhary, \& Thompson-Witrick, 2019; Saremnezhad, Zargarchi, \& Kalantari, 2020). Prebiotic oligosaccharides present favorable organoleptic properties, temperature and acidic stability which make them potential food ingredients (Zhao et al., 2017).

However, new potential prebiotic and less explored oligosaccharides have emerged as possible competitors, namely raffinose. Raffinose is an oligosaccharide composed by galactose, glucose, and fructose residues that can be hydrolyzed to D-galactose and sucrose by the enzyme $\alpha$-galactosidase ( $\alpha$-GAL) (Tester \& Karkalas, 2003). This trisaccharide is non-digestible to humans but can be metabolized by colon bacteria

\footnotetext{
* Corresponding author.

E-mail address: lrmr@deb.uminho.pt (L.R. Rodrigues).
} 
(Fernando et al., 2010), therefore being considered a prebiotic candidate. Furthermore, raffinose can be produced from cheap and abundant agro-residues such as soybean waste, holding a promising environmental and economic advantage.

Prebiotic oligosaccharides are used by the gut microbiota to growth and to produce short-chain fatty acids (SCFAs), lactate (a known precursor of SCFAs) (Flint, Duncan, Scott, \& Louis, 2015), gases (mostly carbon dioxide and hydrogen), among other products (Topping \& Clifton, 2001). The most common SCFAs being produced are acetate, propionate and butyrate, widely reported as key metabolites for intestinal health, benefiting also other body sites than the gut (Gibson et al., 2017).

Different approaches for testing the prebiotic potential of new compounds have been described. However, an in vitro model is considered a more suitable first-stage strategy to screen prebiotics candidates (Date et al., 2014), contrarily to in vivo studies which are expensive and time-consuming.

In agreement with the recent International Scientific Association for Probiotics and Prebiotics (ISAPP) guidelines, the in vitro models should include the use of fecal inocula and accurate high-throughput sequencing techniques to evaluate the microbial modulation. The prebiotic potential of the raffinose family of oligosaccharides has been reported using single or co-cultured microorganisms' models (MartínezVillaluenga, Frías, Vidal-Valverde \& Gómez, 2005; Ose et al., 2018). Nonetheless, in the light of ISAPP guidelines, these approaches cannot mimic the complex diversity of the intestinal microbiota (Gibson et al., 2017). Up to now, studies regarding the prebiotic effect of pure trisaccharide raffinose meeting the ISAPP requirements are scarcely reported. In this study, an in vitro batch culture model was used to evaluate the prebiotic potential of raffinose and compare it with commercial lactulose, using human fecal inocula of two adult healthy donors. Among the most widely studied and commercialized prebiotics, lactulose presents a chemical structure that better resembles raffinose, therefore comprising an interesting comparison model. Next generation sequencing techniques, namely Illumina MiSeq analysis was used to evaluate microbiota diversity. To the authors' knowledge, this is the first study exploring the prebiotic potential of trisaccharide raffinose, used as a pure substrate and evaluated under an experimental approach compatible with the ISAPP guidelines.

\section{Experimental}

\subsection{Materials}

All chemicals and media components used were of analytical grade and purchased from Sigma-Aldrich (St. Louis, MO), including commercial raffinose, unless specified otherwise.

\subsection{Fecal inoculum}

Fecal samples were collected from two healthy human volunteers (Donor 1, coded D1 and donor 2, coded D2), both aged 26, who were on a non-specific Mediterranean diet, had no metabolic and gastrointestinal diseases, were non-smokers and had not received any antibiotics, pre- or probiotic supplements for at least 3 months before the fecal donation. A voluntary informed consent was obtained from the two fecal donors prior to this study. The fecal samples were $1: 10(\mathrm{w} / \mathrm{w})$ diluted in anaerobic phosphate-buffered saline $(0.1 \mathrm{M})$ at $\mathrm{pH} 7.0$ and stored at $4{ }^{\circ} \mathrm{C}$ overnight before the inoculation. The anaerobic conditions were maintained using $100 \% \mathrm{~N}_{2}$ in the headspace.

\subsection{In vitro fermentation of raffinose}

Fermentation experiments were performed using an in vitro static batch culture model, as described by Amorim et al. (2020). Briefly, the fermentations were run in anaerobic conditions at $37{ }^{\circ} \mathrm{C}$ for $48 \mathrm{~h}$. The growth medium at pH 7.0 was composed of: peptone water $(2 \mathrm{~g} / \mathrm{L})$, yeast extract $(2 \mathrm{~g} / \mathrm{L}), \mathrm{NaCl}(0.1 \mathrm{~g} / \mathrm{L}), \mathrm{KH}_{2} \mathrm{PO}_{4}(40 \mathrm{mg} / \mathrm{L}), \mathrm{K}_{2} \mathrm{HPO}_{4}$ $(40 \mathrm{mg} / \mathrm{L}), \mathrm{MgSO}_{4} .7 \mathrm{H}_{2} \mathrm{O}(0.01 \mathrm{~g} / \mathrm{L}), \mathrm{NaHCO}_{3}(2 \mathrm{~g} / \mathrm{L}), \mathrm{CaCl}_{2} \cdot 6 \mathrm{H}_{2} \mathrm{O}$ $(0.01 \mathrm{~g} / \mathrm{L})$, Tween $80(14.8 \mathrm{ml} / \mathrm{L})$, bile salts $(0.5 \mathrm{~g} / \mathrm{L})$, hemin $(5 \mathrm{mg} / \mathrm{L})$, cysteine $\mathrm{HCl}(0.5 \mathrm{~g} / \mathrm{L})$, vitamin $\mathrm{K} 1(74.1 \mu \mathrm{l} / \mathrm{L}), \mathrm{Na}_{2} \mathrm{~S} .9 \mathrm{H}_{2} \mathrm{O}(0.8 \mathrm{mmol} /$ $\mathrm{L})$ and resazurine $(1 \mathrm{mg} / \mathrm{L})$. The raffinose solution was added at a final concentration of $10 \mathrm{~g} / \mathrm{L}$, when required.

The vitamin $\mathrm{k} 1$ and substrate solutions were filter-sterilized and added to the remaining medium components sterilized by autoclave at $121{ }^{\circ} \mathrm{C}$ during $20 \mathrm{~min}$. The fecal inoculum was added to the growth medium at $11 \%(\mathrm{v} / \mathrm{v})$. The anaerobic conditions were attained by pressurizing the bottles headspace with $\mathrm{N}_{2}$ up to $170 \mathrm{KPa}$.

Samples of the headspace and the fermentation broth were collected at different time points. The latter were centrifuged at $4000 \mathrm{x} \mathrm{g}$ for $10 \mathrm{~min}$ and the supernatant was collected and stored at $-20{ }^{\circ} \mathrm{C}$ for further analysis. At $48 \mathrm{~h}$ the final $\mathrm{pH}$ of the medium was measured and the biomass samples were centrifuged, washed and resuspended in PBS $(0.1 \mathrm{M}, \mathrm{pH} 7.0)$, and stored at $-20{ }^{\circ} \mathrm{C}$ for DNA isolation and further sequencing analysis. Fermentations assays were performed in duplicate, running a negative control as blank with no prebiotic addition.

\subsection{Analytical techniques}

The substrate consumption and the production of lactate and SCFAs were analyzed by high performance chromatography (HPLC) according to Amorim et al. (2020). The headspace samples were evaluated by gas chromatography (GC) as described by Arantes, Alves, Alfons, Alves, and Sousa (2017).

\subsection{Microbial analysis}

Liquid samples from fecal inocula and fermentation broth (48 h) were taken and used for DNA extraction using the FastDNA SPIN kit for soil (MP Biomedicals, Solon, OH), according to the manufacturer's procedures. The extracted DNA samples were further submitted to highthroughput sequencing (16S rRNA gene) by Illumina MiSeq technology, as described in detail previously (Salvador et al., 2019). All the sequencing analysis were performed at RTL Genomics (Lubbock, TX) and all the samples were analyzed in duplicate. All the sequences were submitted as FASTQ files at the European Nucleotide Archive under the accession number: ERS3592391, ERS3592398, ERS3592399 and ERS3592411 (BioProject accession number: PRJEB33616).

\subsection{Data analysis}

The negative (blank) and the positive (lactulose) controls reported previously by the same authors (Amorim et al., 2020) were used in this study for comparison purposes. Paired and unpaired t-tests were performed when required. Significant differences were considered when pvalue was $<0.05$.

\section{Results and discussion}

\subsection{Substrate intake and production of lactate and short-chain fatty acids (SCFAs)}

The total production of lactate and SCFAs (acetate, propionate and butyrate), and the residual substrate present in the fermentation medium are shown in Fig. 1. The fermentation of lactulose and raffinose at $10 \mathrm{~g} / \mathrm{L}$ increased significantly the total amount of lactate and SCFAs ( $t$-test student, $\alpha=0.05$ ) for both donors, as consequence of the selective growth-stimulation of SCFAs-producing bacteria (detailed in section 3.4). The results presented in Fig. 1 are in accordance with previous evaluations of the prebiotic effect reported for lactulose, using human fecal inocula (Ito et al., 1997), and raffinose using single cultures of intestinal bacteria (Ose et al., 2018). 

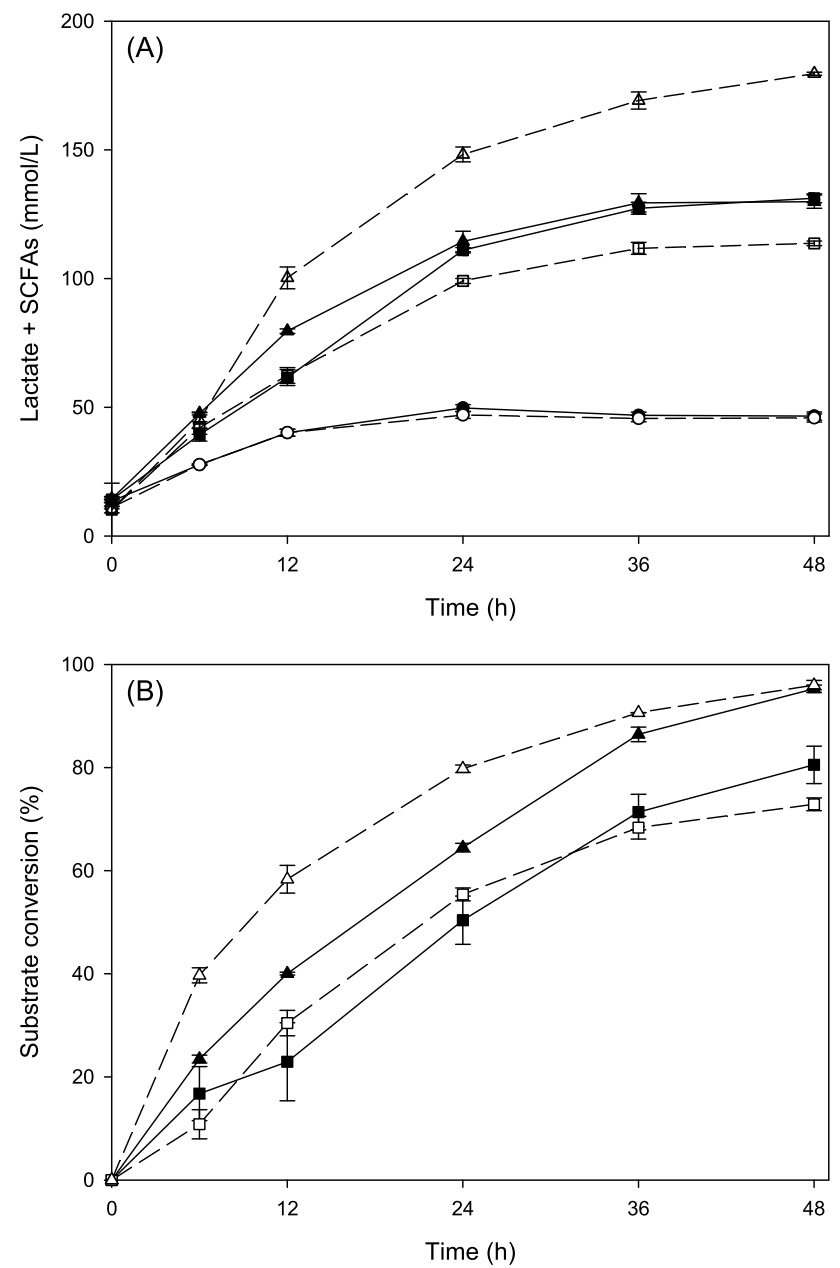

Fig. 1. Total production of lactate and short chain fatty acids (SCFAs) (A) and conversion of lactulose or raffinose (B) supplemented at a starting concentration of $10 \mathrm{~g} / \mathrm{L}$. The fermentations were run during $48 \mathrm{~h}$, using human fecal inocula from donors D1 and D2. A negative control was performed in the absence of prebiotics (blank): Blank D1 (־); Blank D2 (--૦-); Lactulose D1 (——); Lactulose D2 ( $\bullet-$; Raffinose D1 ( —); Raffinose D2 $(-\triangle-)$. Results represent the average of two independent fermentations and the analysis of each sample in triplicate \pm standard deviation.

The maximum production of lactate and SCFAs was achieved at $24 \mathrm{~h}$ for the blank $(49.7 \pm 1.3 \mathrm{mmol} / \mathrm{L} \mathrm{D} 1$, and $47.0 \pm 1.3 \mathrm{mmol} / \mathrm{L} \mathrm{D} 2)$ and between 36 and $48 \mathrm{~h}$ for both lactulose $(131.2 \pm 1.7 \mathrm{mmol} / \mathrm{L} \mathrm{D} 1$ and $113.6 \pm 0.9 \mathrm{mmol} / \mathrm{L} \mathrm{D} 2)$ and raffinose $(129.9 \pm 2.6 \mathrm{mmol} / \mathrm{L} \mathrm{D} 1$ and $179.6 \pm 0.6 \mathrm{mmol} / \mathrm{L} \mathrm{D} 2)$. At this time point, the highest substrate consumption was found (lactulose: $80.5 \pm 3.6 \% \mathrm{D} 1$ and $72.9 \pm 1.2 \%$ D2; raffinose: $95.3 \pm 0.7 \% \mathrm{D} 1$ and $96.0 \pm 0.9 \% \mathrm{D} 2$ ).

Raffinose was mainly consumed during the first $24 \mathrm{~h}$ of fermentation $(64.5 \pm 1.20 .8 \% \mathrm{D} 1$ and $79.8 \pm 0.7 \% \mathrm{D} 2)$, leading to the faster accumulation of lactate and SCFAs $(114.9 \pm 4.0 \mathrm{mmol} / \mathrm{L} \mathrm{D} 1$ and $148.2 \pm 2.9 \mathrm{mmol} / \mathrm{L}$ D2) (Fig. 1). Additionally, when a $10 \mathrm{~g} / \mathrm{L}$ mixture of lactulose and raffinose (1:1) was fermented by the fecal inocula of both donors, raffinose was firstly consumed, thus corroborating the preference of fecal microbiota for this substrate (data not shown).

Interestingly, for D2 the total amount of SCFAs and lactate obtained at $48 \mathrm{~h}$ with raffinose was 1.4 -fold higher when compared with lactulose, while D1 presented no significant differences. This difference may be related with the specific microbiota diversity of each donor inoculum (Table A.1, Appendix A). Adamberg et al. (2018) also reported that fecal microbiota of normal and overweight children exhibited different compositions that responded in specific manners to

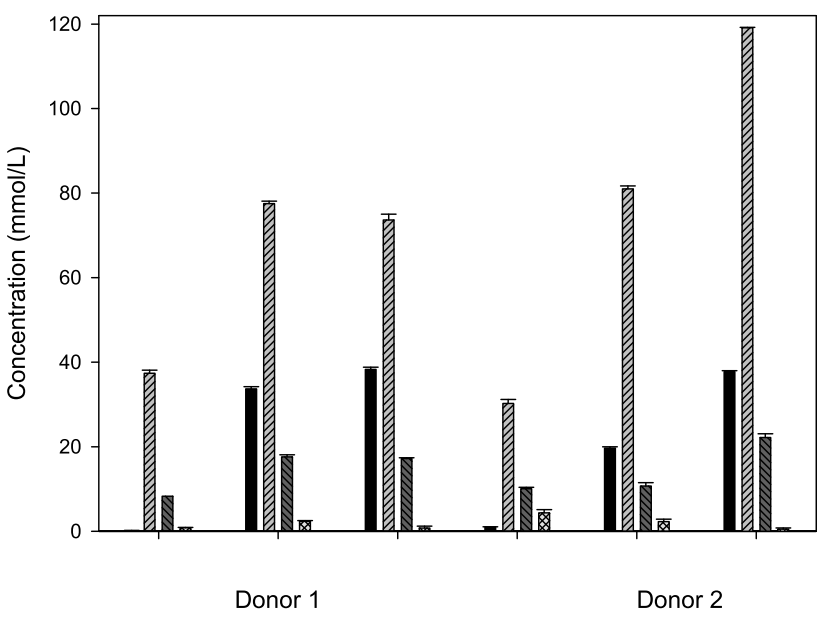

Fig. 2. Production profile of lactate and short chain fatty acids obtained after $48 \mathrm{~h}$ of fermentation, using human fecal inocula from donors D1 and D2. The medium was supplemented with lactulose or raffinose at a starting concentration of $10 \mathrm{~g} / \mathrm{L}$. A negative control was performed in the absence of prebiotics (blank): Lactate ( $\square$ ); Acetate ( घIIIA ); Proprionate (MIIV); Butyrate ( ). Results represent the average of two independent fermentations and the analysis of each sample in triplicate \pm standard deviation.

oligosaccharides and polysaccharides. Particularly, lactic acid was the main fermentation product for overweight children inocula, while the inocula of normal weight children resulted mostly on the production of acetate and butyrate. These facts highlight the need of testing a larger and more representative sample size of donors, including volunteers from different age, culture, health backgrounds and dietary habits, among others.

Besides the total production of SCFAs, the specific profile production, i.e. the proportions in which each SCFA is being produced, is also essential to evaluate the potential benefits on the host health related with the intake of these prebiotics (Gibson et al., 2017).

Fig. 2 shows the respective production profiles of lactate, acetate, propionate and butyrate, obtained at $48 \mathrm{~h}$ of fermentation, using human fecal inocula from D1 and D2.

Overall, acetate was the main SCFAs accumulated at $48 \mathrm{~h}$ (D1: $37.4 \pm 0.7 \mathrm{mmol} / \mathrm{L}$ blank, $77.5 \pm 0.6 \mathrm{mmol} / \mathrm{L}$ lactulose and $73.6 \pm 1.4 \mathrm{mmol} / \mathrm{L}$ rafinose; D2: $30.2 \pm 0.9$ blank, $81.0 \pm 0.7$ lactulose and $119.1 \pm 0.1$ rafinose), with raffinose presenting the highest production of this metabolite that has been reported as playing an important role on colorectal cancer prevention (Casanova, AzevedoSilva, Rodrigues, \& Preto, 2018; Ferro et al., 2016; Oliveira et al., 2015).

On the other hand, lactate was the second most generated metabolite and it was only produced in the presence of a prebiotic substrate, with raffinose presenting the highest values $(38.3 \pm 0.5 \mathrm{mmol} / \mathrm{L} \mathrm{D} 1$ and $37.8 \pm 0.2 \mathrm{mmol} / \mathrm{L} \mathrm{D} 2$ ). These results suggest that both raffinose and lactulose may stimulate the growth of lactate-producing bacteria. Lactate is a known precursor of SCFAs ant it has been reported as holding bioactive properties on human health, namely by being able to regulate critical functions of macrophages and dendritic cells of the immune system and to modulate inflammatory activation of epithelial cells (Garrote, Abraham, \& Rumbo, 2015). Moreover, lactate and lactate-producing bacteria may exert an effect on extra-intestinal sites, such as vagina representing a key line defense against vaginal pathogens (Amabebe \& Anumba, 2018). Thus, raffinose presents potential to be applied on prebiotic formulations for vaginal infections.

Ito et al. (1997) and Ose et al. (2018) also reported acetate and lactate as the most abundant SCFA resulting from the fermentation of lactulose and raffinose using fecal inocula and mono-culture models, 
Table 1

$\mathrm{pH}$ and concentration of ammonia in the fermentation broth after $48 \mathrm{~h}$ of human fecal inocula growth from donors D1 and D2. The absence of prebiotics (blank) and the supplementation of lactulose or raffinose at $10 \mathrm{~g} / \mathrm{L}$ were studied. Results represent the average of two independent fermentations and the analysis of each sample in triplicate \pm standard deviation.

\begin{tabular}{lllll}
\hline Assay & $\mathrm{D} 1$ & \multicolumn{3}{c}{$\mathrm{D} 2$} \\
\cline { 2 - 5 } & $\mathrm{pH}$ & $\begin{array}{l}\text { Ammonia } \\
(\mathrm{mg} / \mathrm{L})\end{array}$ & $\mathrm{pH}$ & $\begin{array}{l}\text { Ammonia } \\
(\mathrm{mg} / \mathrm{L})\end{array}$ \\
\hline Blank & $7.08 \pm 0.02$ & $152 \pm 7$ & $7.00 \pm 0.03$ & $162 \pm 5$ \\
Lactulose & $3.56 \pm 0.03$ & $59 \pm 1$ & $3.585 \pm 0.05$ & $49 \pm 3$ \\
Raffinose & $3.502 \pm 0.002$ & $49 \pm 6$ & $3.605 \pm 0.005$ & $42 \pm 2$ \\
\hline
\end{tabular}

respectively.

The supplementation of lactulose or raffinose did not increase the production of butyrate. However, since lactate is an intermediary of other SCFAs, it would be possible that by increasing the fermentation time, a decrease of this metabolite and subsequent accumulation of SCFAs would be observed. Moreover, Bothe et al. (2017) reported a dose-dependent prebiotic effect of lactulose, which influenced the production of butyrate, using a computer-controlled in vitro model of the human large intestine. This highlights the importance of establishing the most effective prebiotic dose through the use of in vivo models.

\subsection{Variation of $p H$ and ammonia concentration}

The increased production of SCFA due to the prebiotic fermentation causes a $\mathrm{pH}$ drop, which in turn promotes the reduction of intestinal pathogenic agents and ammonia production that is related with bad fecal odor and colon carcinogenesis (Visek, 1978).

The $\mathrm{pH}$ and the ammonia concentration values obtained after $48 \mathrm{~h}$ of fermentation are presented in Table 1 . As expected, the supplementation with lactulose and raffinose led to an equivalent $\mathrm{pH}$ and ammonia reduction. Adamberg et al. (2018) also reported a reduction of the final $\mathrm{pH}$ values from 7.3 to 7.4 (control cultures) to $\mathrm{pH} 5.1$ and 5.3 (cultures supplemented with a mixture of raffinose and melibiose).

Compared with in vivo and human studies, the in vitro models present some limitations, since they cannot replicate all the complex conditions occurring in the colon (Poeker et al., 2018). For instance, in an in vitro model without $\mathrm{pH}$ control, the fermentation medium mimics the distal colon $\mathrm{pH}$ at baseline. Nonetheless, the SCFAs formed during the fermentation are not absorbed, as it occurs using an in vivo model, thus, these metabolites cause significant variations of the medium $\mathrm{pH}$ (Pham \& Mohajeri, 2018), which is a limitation of this type of models. On the other hand, the rapid absorption of SCFAs in in vivo models limits their measurement (Carlson, Erickson, Hess, Gould, \& Slavin, 2017). Therefore, the in vitro models are an attractive alternative tool to study the kinetics of colonic fermentation (Tsitko, Mattila, Maukonen, \& Saarela, 2019), but they should be regarded as semi-representative models of colonic fermentation.

\subsection{Production of $\mathrm{H}_{2}$ and $\mathrm{CO}_{2}$}

The prebiotic fermentation by human fecal inocula also exerts an effect on the production of gases. For both donors (D1 and D2), the addition of raffinose increased significantly the production of $\mathrm{CO}_{2}$ when compared with the blank in particular for D1 (Fig. 3). The maximum production of $\mathrm{CO}_{2}$ obtained with raffinose was $10.8 \pm 0.8 \mathrm{mmol} /$ $\mathrm{L}_{\text {medium }}$ at $48 \mathrm{~h}$ (D1) and $5.2 \pm 0.3 \mathrm{mmol} / \mathrm{L}_{\text {medium }}$ at $36 \mathrm{~h}$ (D2).

Production of methane was not detected, which may suggest the absence of methanogenic bacteria in the original microbiota. In fact, it is known that only a fraction of the human population harbor methanogenic bacteria on their gut microbiota (Ghoddusi, Grandison,
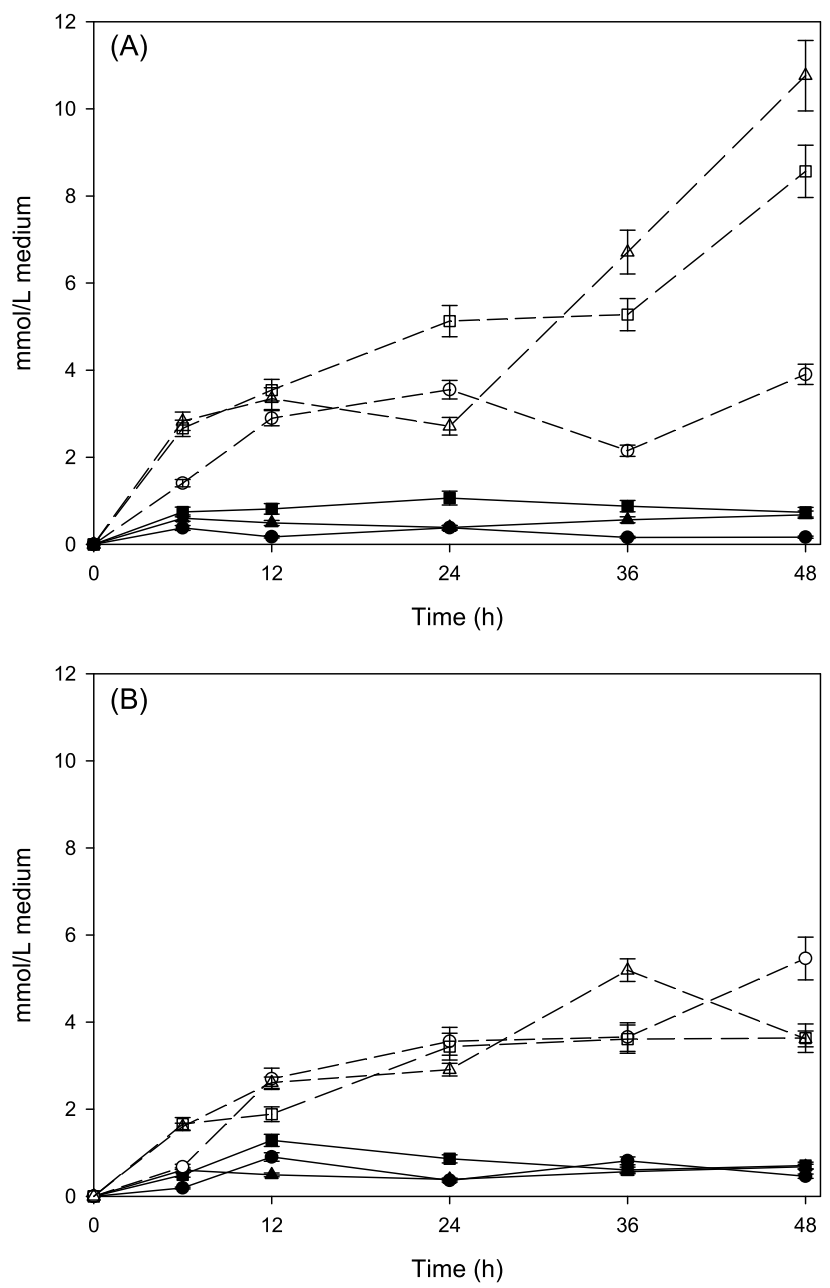

Fig. 3. Production of $\mathrm{H}_{2}$ and $\mathrm{CO}_{2}$ by human fecal inocula from donors D1 (A) and D2 (B) during $48 \mathrm{~h}$. The absence of prebiotics (blank) and the supplementation of lactulose or raffinose at $10 \mathrm{~g} / \mathrm{L}$ were studied: Blank $\mathrm{H}_{2}(\longrightarrow-)$; Blank $\mathrm{CO}_{2}(--\mathrm{O}-)$; Lactulose $\mathrm{H}_{2}(--)$; Lactulose $\mathrm{CO}_{2}(-\square-)$; Raffinose $\mathrm{H}_{2}(-)$; Raffinose $\mathrm{CO}_{2}(-\Delta-)$. Results represent the average of two independent fermentations and the analysis of each sample in triplicate \pm standard deviation.

Grandison, \& Tuohy, 2007). However, the DNA profiles of the fermentation samples must be analyzed to support this hypothesis as further described in section 3.4.

Excessive production of gas resulting from raffinose fermentation may cause bloating and flatulence problems, which represents a clinical disincentive to its use (Cummings, Macfarlane, \& Englyst, 2001). Dahl, Hanifi, Zello, and Tyler (2014) reported a slight increase of the flatulence and bloating rate due to the daily consumption of $5 \mathrm{~g}$ of raffinose by healthy adults, but no differences were found for diarrhea or abdominal pain when compared with the control. Suarez et al. (1999) verified an increase of the flatus rate in human subjects caused by the ingestion of $80 \mathrm{~g}$ of different soybean flours derived from beans. In particular, the flour containing higher amounts of raffinose $(0.51 \%)$ resulted in the highest flatus rate. Therefore, it is essential to determine the proper dose of raffinose intake through in vivo models, consisting in the recommended dose that maximizes the prebiotic effect, while presenting minimal secondary effects.

\subsection{Effect of prebiotics fermentation on intestinal microbiota}

The microbiota analysis of the fecal inocula showed the 

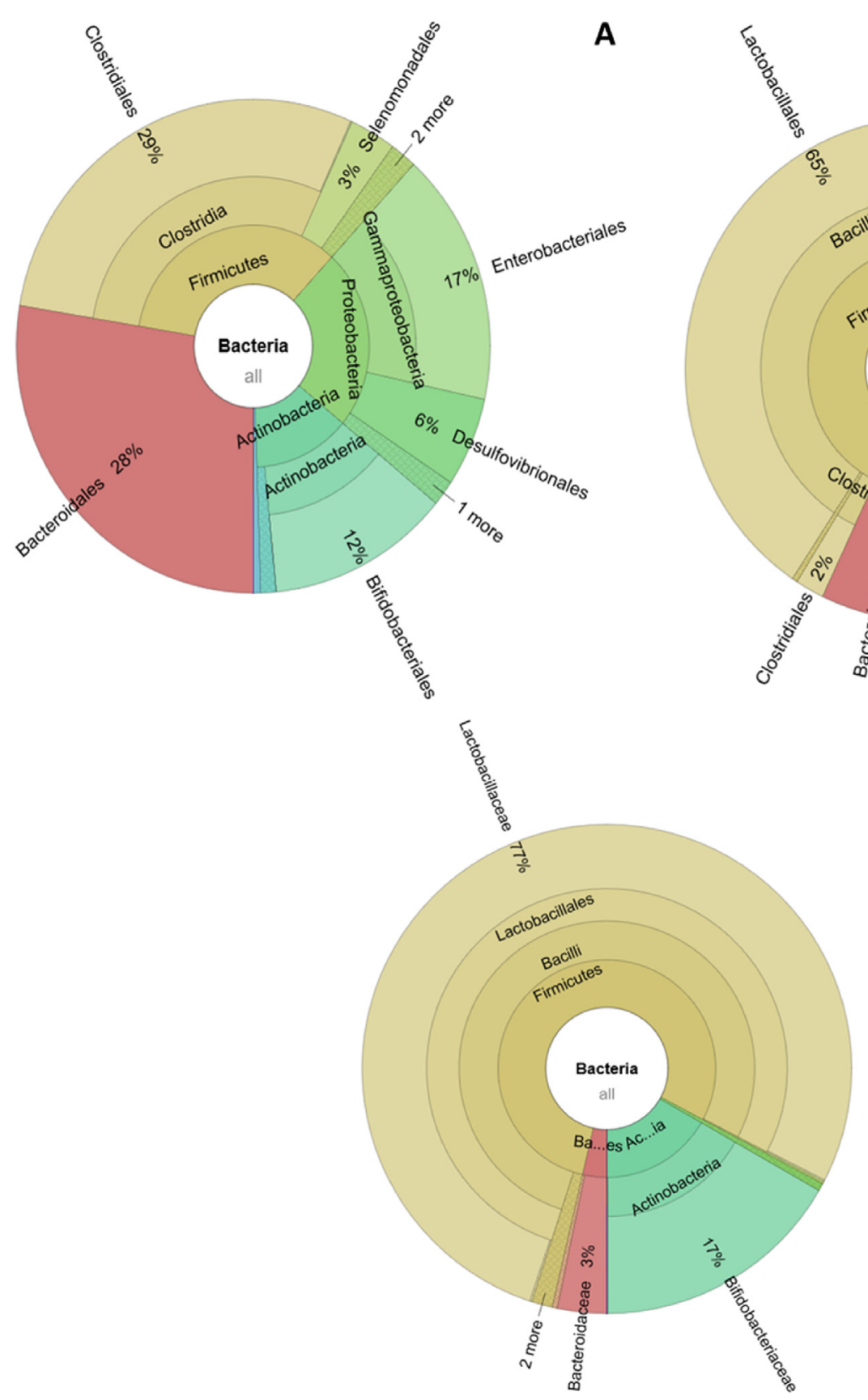

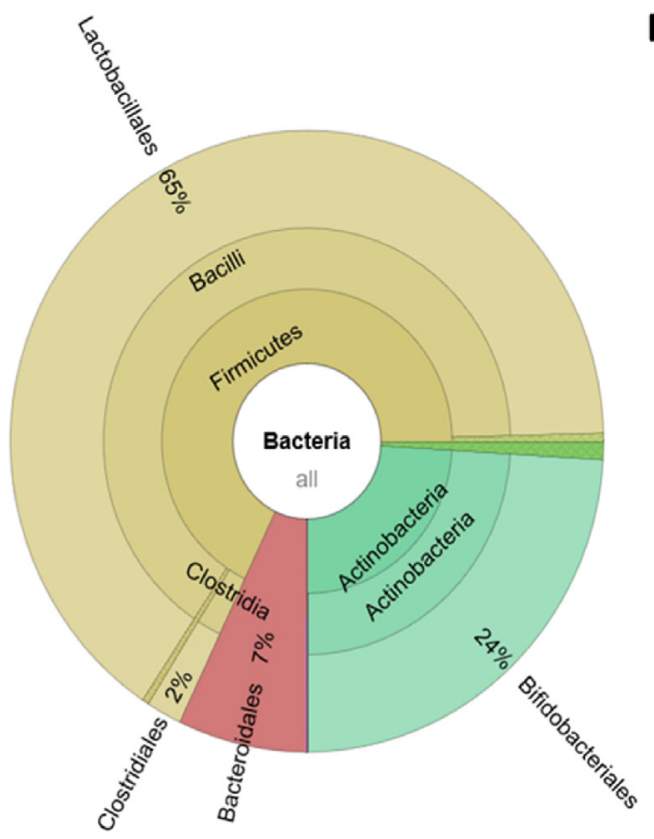

C

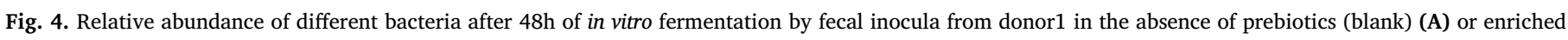
with a prebiotic solution of lactulose (B) or raffinose (C) at $10 \mathrm{~g} / \mathrm{L}$.

characteristic gut microbiota diversity of healthy human adults (Eckburg et al., 2005), including the presence of six bacterial phyla, of which Firmicutes dominate in terms of relative abundance, Table A.1, Appendix A.

The medium supplementation with lactulose or raffinose resulted in a similar modulation of the gut microbiota of both donors after $48 \mathrm{~h}$ of fermentation, Fig. 4 and Fig. 5. When compared with the blank, both prebiotics increased the relative abundance of well-known probiotics, namely organisms belonging to Bifidobacteria and Lactobacillus, with raffinose showing a $1.8 \pm 0.8$ - and $4.1 \pm 0.1$-fold increase of Bifidobacteria and $725 \pm 45$ - and $750 \pm 0$-fold increase of Lactobacillus for donors 1 and 2, respectively.

However, after the fermentation of lactulose or raffinose, microorganisms belonging to the order Lactobacillales presented the highest relative abundance for D1, while Bifidobacteriales dominated in D2. Those differences could be explained by the intrinsic variations on the original inocula of the donors. Therefore, the specific prebiotic effect will greatly depend on the original inocula of the donors, although the main pattern of bacterial growth generated by the prebiotic fermentation were comparable between them. This fact highlights, as previously mentioned, the importance of performing in vitro and in vivo studies with a representative sample, in terms of size and diversity of subjects.

Lactobacillus and Bifidobacterium are widely known probiotic able to use complex carbohydrates and to mainly produce acetate and lactate, releasing $\mathrm{CO}_{2}$, which corroborates the results presented in sections 3.1 and 3.2. However, these bacteria may also stimulate butyrate-producing microorganisms in case of cross-feeding effect, resulting in the production of this SCFA (Gibson et al., 2017). 


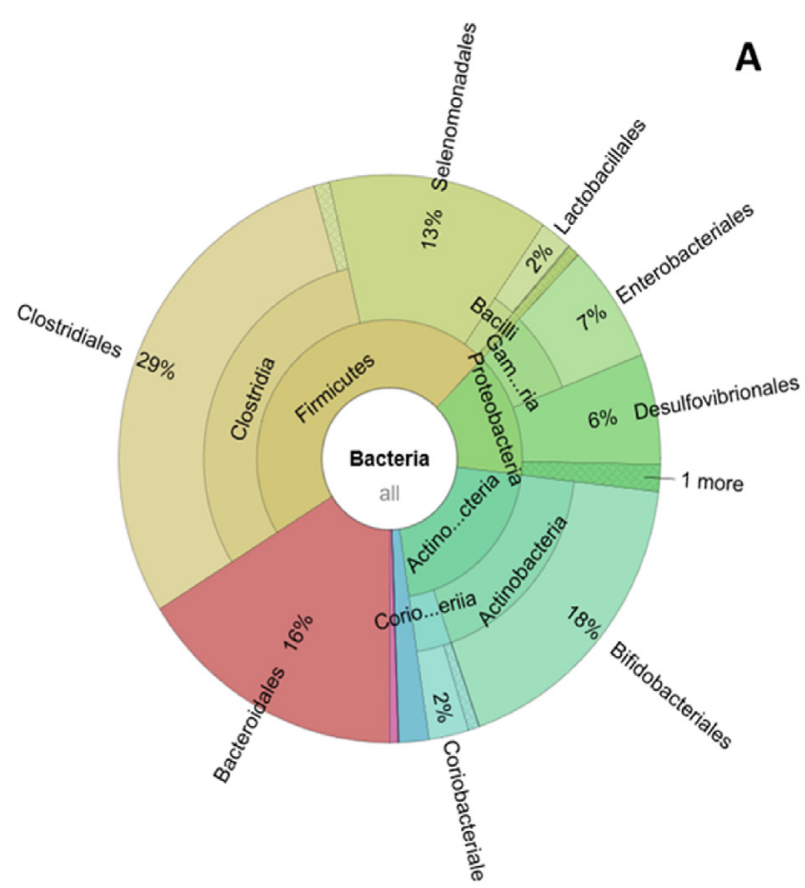

A

B

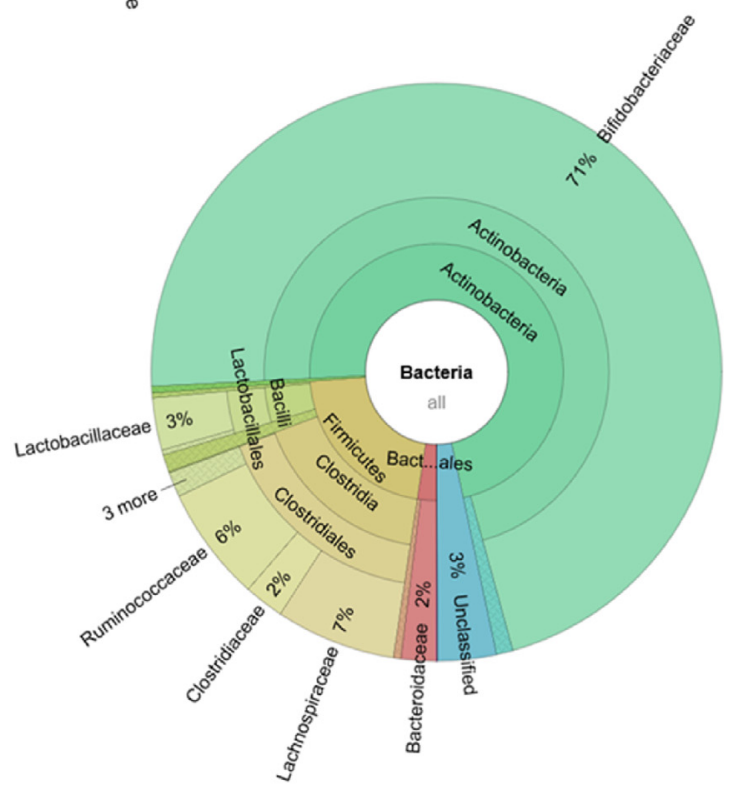

C

Fig. 5. Relative abundance of different bacteria after $48 \mathrm{~h}$ of in vitro fermentation by fecal inocula from donor 2 in the absence of prebiotics (blank) (A) or enriched with a prebiotic solution of lactulose (B) or raffinose (C) at $10 \mathrm{~g} / \mathrm{L}$.

On the other hand, a significant reduction of relative amount of Proteobacteria was observed due to the fermentation of prebiotics. The highest reduction was achieved for raffinose $(53 \pm 7$-fold D1, $43 \pm 8$ fold D2).

The abundance of Proteobacteria on gut microbiota is associated to several intestinal diseases. This phylum comprises several known human pathogens (Rizzatti, Lopetuso, Gibiino, Binda, \& Gasbarrini, 2017), including Escherichia coli whose relative abundance was significantly reduced by de addition of lactulose (32.0 \pm 5 -fold D1, $1350 \pm 650$-fold D2) and raffinose (12 \pm 4-fold D1, $28 \pm 8$-fold D2) (Table A.1, Appendix A). The reduction in the ammonia concentration (section 3.3) was confirmed by the reduction of ammonia producing bacteria including $E$. coli and Clostridium sp.

The absence of methanogenic archaea on the original inoculum of D1 could explain the lack of $\mathrm{CH} 4$ production (Table A.1, Appendix A). However, D2 presents Methanobacteriacea members on its original inoculum $(0.85 \pm 0.15 \%)$ which in turn decreased with the fermentation of lactulose and raffinose, with raffinose presenting the most significant reduction ( $25 \pm 5$-fold). Nevertheless, the production of CH4 from D2 inoculum could be below the sensitivity limit of the GC method, since the relative abundance of these bacteria was significantly low on both inoculum and blank samples.

Taking into account the results herein gathered, raffinose presents a high prebiotic potential, comparable to the well-accepted lactulose, specially to act on Bifidobacterium and Lactobacillus reduced dysbiosis.

\section{Conclusions}

The addition of trisaccharide raffinose increased the production of SCFAs and $\mathrm{CO}_{2}$, reducing the final $\mathrm{pH}$ of the medium and the ammonia concentration. Moreover, the presence of raffinose as substrate increased the relative abundance of Bifidobacterium and Lactobacillus species, while reducing Proteobacteria, including widely known pathogens, such as E. coli. 
This study strongly suggests that raffinose holds potential prebiotic properties for human health and, subsequently, potential to be commercialized as functional food ingredient. Nevertheless, in vivo studies should be further carried out to access its prebiotic effect in a more realistic model and to establish a more accurate recommended daily dose of this compound.

\section{CRediT authorship contribution statement}

Cláudia Amorim: Investigation, Methodology, Writing - original draft. Sara C. Silvério: Investigation, Methodology, Writing - review \& editing. Beatriz B. Cardoso: Investigation, Methodology. Joana I. Alves: Investigation, Methodology, Writing - review \& editing. Maria Alcina Pereira: Funding acquisition, Resources, Validation, Writing review \& editing. Lígia R. Rodrigues: Funding acquisition, Resources, Validation, Supervision, Writing - review \& editing, Project administration.

\section{Declaration of competing interest}

None.

\section{Acknowledgments}

CA an BBC acknowledge her grants (UMINHO/BPD/4/2019 and SFRH/BD/132324/2017) from Portuguese Foundation for Science and Technology (FCT). The study received financial support from FCT under the scope of the strategic funding of UID/BIO/04469/2020 unit; COMPETE 2020 (POCI-01-0145-FEDER-006684), through national funds and where applicable co-financed by the FEDER, within the PT2020 Partnership Agreement; the Projects FoSynBio (POCI-01-0145FEDER-029549) and NewFood - Food Technologies Valorization (NORTE-324 01-0246-FEDER-000043). The authors also acknowledge BioTecNorte operation (NORTE-01-0145-FEDER-000004) funded by the European Regional Development Fund under the scope of Norte2020 -Programa Operacional Regional do Norte.

\section{Appendix A. Supplementary data}

Supplementary data to this article can be found online at https:// doi.org/10.1016/j.lwt.2020.109322.

\section{References}

Adamberg, K., Adamberg, S., Ernits, K., Larionova, A., Voor, T., Jaagura, M., et al. (2018). Composition and metabolism of fecal microbiota from normal and overweight children are differentially affected by melibiose, raffinose and raffinose-derived fructans. Anaerobe, 52, 100-110.

Adamberg, S., Sumeri, I., Uusna, R., Ambalam, P., Kondepudi, K. K., Adamberg, K., et al. (2014). Survival and synergistic growth of mixed cultures of bifidobacteria and lactobacilli combined with prebiotic oligosaccharides in a gastrointestinal tract simulator. Microbial Ecology in Health and Disease, 25, 1-9.

Amabebe, E., \& Anumba, D. O. C. (2018). The vaginal microenvironment: The physiologic role of lactobacilli. Frontiers of Medicine, 5.

Amorim, C., Silvério, S. C., Cardoso, B. B., Alves, J. I., Pereira, M. A., \& Rodrigues, L. R. (2020). In vitro assessment of prebiotic properties of xylooligosaccharides produced by Bacillus subtilis 3610 in a single-step process. Carbohydrate Polymers, 229, 115460.

Arantes, A. L., Alves, J. I., Alfons, J. M., Alves, M. M., \& Sousa, D. Z. (2017). Enrichment of syngas-converting communities from a multi-orifice baffled bioreactor. Microbial Biotechnology, 11, 639-646.

Bothe, M., Maathuis, A., Bellmann, S., van der Vossen, J., Berressem, D., Koehler, A., et al. (2017). Dose-dependent prebiotic effect of lactulose in a computer-controlled in vitro model of the human large intestine. Nutrients, 9, 767.

Carlson, J. L., Erickson, J. M., Hess, J. M., Gould, T. J., \& Slavin, J. L. (2017). Prebiotic dietary fiber and gut health: Comparing the in vitro fermentations of beta-glucan, inulin and xylooligosaccharide. Nutrients, 9, 1361.

Casanova, M. R., Azevedo-Silva, J., Rodrigues, L. R., \& Preto, A. (2018). Colorectal cancer cells increase the production of short chain fatty acids by Propionibacterium freudenreichii impacting on cancer cells survival. Frontiers in Nutrition, 5, 44.

Cummings, J. H., Macfarlane, G. T., \& Englyst, H. N. (2001). Prebiotic digestion and fermentation. American Journal of Clinical Nutrition, 73, 415s-420s.

Dahl, W. J., Hanifi, A., Zello, G. A., \& Tyler, R. T. (2014). Gastrointestinal tolerance to daily canned chickpea intake. Canadian Journal of Dietetic Practice and Research, 75, 218-221.

Das, K., Choudhary, R., \& Thompson-Witrick, K. A. (2019). Effects of new technology on the current manufacturing process of yogurt to increase the overall marketability of yogurt. Lebensmittel-Wissenschaft und -Technologie- Food Science and Technology, 108, 69-80.

Date, Y., Nakanishi, Y., Fukuda, S., Nuijima, Y., Kato, T., Umehara, M., et al. (2014). In vitro evaluation method for screening of candidate prebiotic foods. Food Chemistry, $152,251-260$.

Eckburg, P. B., Bik, E. M., Bernstein, C. N., Purdom, E., Dethlefsen, L., Sargent, M., et al. (2005). Diversity of the human intestinal microbial flora. Science, 308, 1635-1638.

Fernando, W. M. U., Hill, J. E. G., Zello, A., Tyler, R. T., Dahl, W. J., \& Van Kessel, A. G. (2010). Diets supplemented with chickpea or its main oligosaccharide component raffinose modify faecal microbial composition in healthy adults. Beneficial Microbes, 1, 197-207.

Ferro, S., Azevedo-Silva, J., Casal, M., Côrte-Real, M., Baltazar, F., \& Preto, A. (2016). Characterization of acetate transport in colorectal cancer cells and potential therapeutic implications. Oncotarget, 7, 70639-70653.

Flint, H. J., Duncan, S. H., Scott, K. P., \& Louis, P. (2015). Conference on 'diet, gut microbiology and human health' symposium 3: Diet and gut metabolism: Linking microbiota to beneficial products of fermentation links between diet, gut microbiota composition and gut metabolism. Proceedings of the Nutrition Society, 74, 13-22.

Forbes (2015). Consumers want healthy foods-and will pay more for them. https://www. forbes.com/sites/nancygagliardi/2015/02/18/consumers-want-healthy-foods-andwill-pay-more-for-them/\#24de393275c5, Accessed date: 31 January 2020.

Garrote, G. L., Abraham, A. G., \& Rumbo, M. (2015). Is lactate an undervalued functional component of fermented food products? Frontiers in Microbiology, 6.

Ghoddusi, H. B., Grandison, M. A., Grandison, A. S., \& Tuohy, K. M. (2007). In vitro study on gas generation and prebiotic effects of some carbohydrates and their mixtures. Anaerobe, 13, 193-199.

Gibson, G. R., Hutkins, R., Sanders, M. E., Prescott, S. L., Reimer, R. A., Salminen, S. J., et al. (2017). The International Scientific Association for Probiotics and Prebiotics (ISAPP) consensus statement on the definition and scope of prebiotics. Nature Reviews Gastroenterology \& Hepatology, 14, 491-502.

Grand View Research (2018). Prebiotics market size, share \& trends analysis by ingredients (fos, inulin, gos, mos), by application (food and beverages, dietary supplements, animal feed), by region, and segment forecasts, 2014 - 2024. https://www. grandviewresearch.com/industry-analysis/prebiotics-market, Accessed date: 31 January 2020.

Ito, Y., Moriwaki, H., Muto, Y., Kato, N., Watanabe, K., \& Ueno, K. (1997). Effect of lactulose on short-chain fatty acids and lactate production and on the growth-of faecal flora, with special reference to Clostridium difficile. Journal of Medical Microbiology, 46, 80-84.

Kang, D.-W., Adams, J. B., Coleman, D. M., Pollard, E. L., Maldonado, J., McDonoughMeans, S., et al. (2019). Long-term benefit of Microbiota Transfer Therapy on autism symptoms and gut microbiota. Scientific Reports, 9.

Martínez-Villaluenga, C., Frías, J., Vidal-Valverde, C., \& Gómez, R. (2005). Raffinose family of oligosaccharides from lupin seeds as prebiotics: Application in dairy products. Journal of Food Protection, 68, 1246-1252.

Oliveira, C. S. F., Pereira, H., Alves, S., Castro, L., Baltazar, F., Chaves, S. R., et al. (2015). Cathepsin D protects colorectal cancer cells from acetate-induced apoptosis through autophagy-independent degradation of damaged mitochondria. Cell Death \& Disease, 6, e1788.

Ose, R., Hirano, K., Maeno, S., Nakagawa, J., Salminen, S., Tochio, T., et al. (2018). The ability of human intestinal anaerobes to metabolize different oligosaccharides: Novel means for microbiota modulation? Anaerobe, 51, 110-119.

Pham, V. T., \& Mohajeri, M. H. (2018). The application of in vitro human intestinal models on the screening and development of pre- and probiotics. Beneficial Microbes, 9, 725-742.

Poeker, S. A., Geirnaert, A., Berchtold, L., Greppi, A., Krych, L., Steinert, R. E., et al. (2018). Understanding the prebiotic potential of different dietary fibers using an in vitro continuous adult fermentation model (PolyFermS). Scientific Reports, 8, 4318.

Rizzatti, G., Lopetuso, L. R., Gibiino, G., Binda, C., \& Gasbarrini, A. (2017). Proteobacteria: A common factor in human diseases. BioMed Research International, 1-7 ID 9351507.

Saad, N., Delattre, C., Urdaci, M., Schmitter, J. M., \& Bressollier, P. (2013). An overview of the last advances in probiotic and prebiotic field. Lebensmittel-Wissenschaft und -Technologie- Food Science and Technology, 50, 1-16.

Salvador, A. F., Cavaleiro, A. J., Paulo, A. M. S., Silva, S. A., Guedes, A. P., Pereira, M. A. et al. (2019). Inhibition studies with 2-bromoethanesulfonate reveal a novel syntrophic relationship in anaerobic oleate degradation. Applied and Environmental Microbiology, 85 e01733-18.

Samanta, A. K., Jayapal, N., Jayaram, C., Roy, S., Kolte, A. P., Senani, S., et al. (2015). Xylooligosaccharides as prebiotics from agricultural by-products: Production and applications. Bioactive Carbohydrates and Dietary Fibre, 5, 62-71.

Saremnezhad, S., Zargarchi, S., \& Kalantari, Z. N. (2020). Calcium fortification of prebiotic ice-cream. Lebensmittel-Wissenschaft und -Technologie, 120, 108890.

Suarez, F. L., Springfield, J., Furne, J. K., Lohrmann, T. T., Kerr, P. S., \& Levitt, M. D. (1999). Gas production in humans ingesting a soybean flour derived from beans naturally low in oligosaccharides. American Journal of Clinical Nutrition, 69, 135-139.

Tester, R. F., \& Karkalas, J. (2003). Carbohydrates: Classification and properties. In B. Caballero, L. Trugo, \& P. M. Finglas (Eds.). Encyclopedia of food sciences and nutrition (pp. 862-875). San Diego: Academic Press.

Topping, D. L., \& Clifton, P. M. (2001). Short-chain fatty acids and human colonic function: Roles of resistant starch and nonstarch polysaccharides. Physiological Reviews, 81, 1031-1064. 
Tsitko, Wiik-Miettinen, Mattila, Rosa-Sibakov, Maukonen, Nordlund, \& Saarela (2019). A small in vitro fermentation model for screening the gut microbiota effects of different fiber preparations. International Journal of Molecular Sciences, 20, 1925.

Visek, W. J. (1978). Diet and cell growth modulation by ammonia. American Journal of Clinical Nutrition, 31, S216-S220.

WHO - World Health Organization (2018). Global report on diabetes. https://apps.who. int/iris/bitstream/handle/10665/204871/9789241565257_eng.pdf.jsessionid = AB2C916A6DBA6120F68D15F7A47BF5BC? sequence =1, Accessed date: 31 January
2020

Yeung, A. W. K., Mocan, A., \& Atanasov, A. G. (2018), Let food be thy medicine and medicine be thy food: A bibliometric analysis of the most cited papers focusing on nutraceuticals and functional foods. Food Chemistry, 269, 455-465.

Zhao, C., Wu, Y., Liu, X., Liu, B., Cao, H., Yu, H., et al. (2017). Functional properties, structural studies and chemo-enzymatic synthesis of oligosaccharides. Trends in Food Science \& Technology, 66, 135-145. 